\title{
DEPRESSÃO: PONTOS DE VISTA E CONHECIMENTO DE ENFERMEIROS DA
} REDE BÁSICA DE SAÚDE ${ }^{1}$

\author{
Mariluci Camargo Ferreira da Silva ${ }^{2}$ \\ Antonia Regina Ferreira Furegato ${ }^{3}$ \\ Moacyr Lobo da Costa Júnior ${ }^{4}$
}

Silva MCF, Furegato ARF, Costa ML Júnior. Depressão: pontos de vista e conhecimento de enfermeiros da rede básica de saúde. Rev Latino-am Enfermagem 2003 janeiro-fevereiro; 11(1):7-13.

Objetivou-se identificar os pontos de vista e o conhecimento sobre depressão de enfermeiros da rede básica de saúde. Os 73 participantes que responderam a dois questionários auto-aplicativos, encontraram-se distribuídos em 28 unidades de saúde. Nos resultados gerais dos pontos de vista e do conhecimento, a maioria das respostas estão de acordo com o que se esperava, com médio conhecimento sobre depressão. Entretanto, nas análises individuais dessas questões, os resultados indicam que esses profissionais não estão em contato direto e não sabem identificar pacientes deprimidos, não observam estes indicadores nos pacientes por eles atendidos ou não entendem que seja sua tarefa fazer essa identificação. Surgiram reflexões acerca da possível relação entre as ações de saúde mental com a sua formação profissional. Dessa maneira, o ensino na graduação e a educação continuada passam a ter maior importância no desenvolvimento das ações de enfermagem para a saúde mental.

DESCRITORES: enfermagem, saúde mental, depressão

\section{DEPRESSION: VIEWPOINTS AND KNOWLEDGE OF NURSES FROM THE BASIC HEALTH NETWORK}

This study aimed at identifying the viewpoints and the knowledge on depression of nurses working in the basic health network. The 73 participants who answered two self-applicable questionnaires were distributed in 28 health units. The general results concerning their viewpoints and knowledge are, in most answers, in accordance with what was expected and showed average knowledge related to depression. However, the individual analysis of such answers indicate that these professionals are not in direct contact with and do not know how to identify depressed patients. They do not observe these indicators in the patients that they assist or do not understand that it is their task to do so. Reflections appeared concerning the possible relationship between mental health actions and their professional education. In this way, undergraduate and continuing education become more important in the development of nursing actions for mental health.

DESCRIPTORS: nursing, mental health, depression

\section{DEPRESIÓN: PUNTOS DE VISTA Y CONOCIMIENTO DE ENFERMEROS DE LA RED BASICA DE SALUd}

Se buscó identificar los puntos de vista y el conocimiento de enfermeros de la Red Básica de Salud sobre la depresión. Los 73 participantes estaban distribuidos en 28 unidades de salud y respondieron dos cuestionarios autoaplicativos. Los resultados generales están de acuerdo con lo que se esperaba, o sea, un conocimiento medio sobre depresión. Sin embargo, los análisis individuales de estos aspectos indican que los profesionales no están en contacto directo y no saben identificar pacientes deprimidos, no observan estos indicadores en los pacientes o no comprenden que su tarea sea realizar esta identificación. Surgieron reflexiones acerca de la posible relación entre las acciones de salud mental con su formación profesional. De esta manera, la enseñanza en pregrado y la educación continuada pasan a tener mayor importancia en el desarrollo de las acciones de enfermería para la salud mental.

DESCRIPTORES: enfermería, salud mental, depresión

\footnotetext{
${ }^{1}$ Parte dos resultados da Dissertação de Mestrado apresentada à Escola de Enfermagem de Ribeirão Preto da Universidade de São Paulo; ${ }^{2}$ Doutoranda; ${ }^{3}$ Professor Titular, e-mail: furegato@eerp.usp.br; ${ }^{4}$ Professor Doutor. Escola de Enfermagem de Ribeirão Preto da Universidade de São Paulo, Centro Colaborador da OMS para o desenvolvimento da pesquisa em enfermagem
} 
INTRODUÇÃO

$\boldsymbol{A}$ rede básica de saúde constitui-se na principal porta de entrada das pessoas na busca da atenção à sua saúde, incluindo aquelas em sofrimento psíquico ou portadores de transtorno mental.

A depressão é, reconhecidamente, um problema de saúde pública. É um dos processos patológicos com maior freqüência na atenção primária médica, com (cerca de $10 \%$ de todas as novas consultas). Afeta a população em geral, sendo altamente incapacitante e interferindo de modo decisivo e intenso na vida pessoal, profissional, social e econômica dos portadores. É potencialmente letal, pois, em casos graves, existe o risco contínuo de suicídio. Muitas pessoas sofrem em silêncio, seja porque não consultam, seja porque os profissionais não diagnosticam, nem tratam adequadamente. É fator de risco para outras enfermidades visto que as formas moderadas de depressão podem se apresentar mascaradas por outras queixas, tais como dor de cabeça persistente, dispepsia, falta de apetite, constipação, gosto ruim na boca, gerando altos custos para o sistema de saúde e para a sociedade ${ }^{(1-2)}$.

Durante toda a vida, 31 a $50 \%$ da população brasileira apresentam, pelo menos, um episódio de transtorno mental e cerca de 20 a $40 \%$ necessitam, por conta desses transtornos, de algum tipo de ajuda profissional $^{(3)}$.

As pessoas deprimidas podem não procurar o médico devido aos próprios sintomas causadores da enfermidade, como a falta de energia, indecisão, insegurança e culpabilidade. Além do estigma associado à enfermidade mental, verifica-se a falta de atenção dos profissionais de saúde aos problemas emocionais ${ }^{(1)}$.

Depressão é um transtorno do humor. Muitas vezes, os termos depressão e transtornos afetivos são utilizados como equivalentes. Em seu sentido exato não são. Os transtornos afetivos compõem uma categoria ampla de estados de ânimo (dificuldades no campo das emoções, na capacidade cognitiva, no comportamento e na regularidade das funções corporais). A depressão é a forma mais comum de transtornos afetivos ${ }^{(1)}$.

$\mathrm{Na}$ literatura, encontram-se descritos os seguintes tipos de depressão: transtorno depressivo maior, distimia, depressão integrante do transtorno bipolar I e ll e depressão, como parte da ciclotimia. Os tipos mais comuns são o transtorno depressivo maior e o transtorno bipolar ${ }^{(3-6)}$.

Com base no DSM-IV ${ }^{(7)}$ e CID-10 $0^{(8)}$, os sintomas clínicos da depressão são humor depressivo, tristeza, perda de interesse ou prazer, perda ou ganho de peso significativo, insônia (no início, na metade ou no final do sono) ou hipersonia, agitação ou retardo psicomotor, fadiga ou perda de energia, sentimento de inutilidade ou culpa excessiva ou inadequada, indecisão ou capacidade diminuída de pensar ou concentrar-se e pensamentos de morte recorrentes.

O portador de sintomas depressivos está presente em todos os lugares da comunidade, como escolas, clínicas, hospitais, além dos centros de saúde mental. Dessa forma, todos os enfermeiros necessitam de conhecimentos de enfermagem psiquiátrica para dar cuidados adequados àqueles que apresentam problemas emocionais.

O enfermeiro, em todos os tipos de instituição de saúde, tem a responsabilidade de reconhecer e intervir apropriadamente nos casos em que o indivíduo está sofrendo de um transtorno de humor ${ }^{(9-10)}$.

O enfermeiro ocupa posição singular nos serviços de saúde, pois, geralmente, ele é o primeiro profissional que tem contato com a pessoa que busca atenção nesses serviços. As razões para que o pessoal de enfermagem se ocupe dos transtornos afetivos estão relacionadas com sua experiência e seu preparo profissional. Além disso, os pacientes sentem-se menos intimidados pelos enfermeiros do que por outros agentes de saúde e os aceitam mais facilmente. A assistência de enfermagem não se limita em ajudar o paciente, mas também orientar a família e a comunidade ${ }^{(1)}$.

Por outro lado, os trabalhadores da saúde, inclusive o pessoal de enfermagem, referem que não têm tido acesso à informação atualizada para incorporar os avanços científicos em suas práticas clínicas, nem têm incorporadas as transformações da assistência em saúde mental $^{(1)}$.

Na década de 70, já se dizia que a saúde mental é um componente essencial da saúde, um direito humano, o que vem reforçar a idéia de que é um dever do enfermeiro promover a saúde mental das pessoas ${ }^{(11)}$.

Por entender que o cuidado ao portador de transtorno mental está sempre presente na vida profissional do enfermeiro, independente da sua área de atuação e por considerar que os serviços básicos de saúde são locais de detectação precoce e ação preventiva aos transtornos, decidimos investigar o conhecimento e a opinião do enfermeiro sobre depressão, com vistas a formular propostas para resolução desta problemática. 
OBJETIVO

Identificar os pontos de vista e o conhecimento de enfermeiros, da rede básica de saúde, sobre depressão.

\section{MATERIAL E MÉTODO}

A abordagem quantitativa descritiva foi escolhida tendo em vista o tipo de coleta de dados. Utilizou-se a psicometria que abarca os testes psicológicos, os inventários, os questionários e as escalas ${ }^{(12)}$.

Local: a presente pesquisa foi realizada em 28 serviços de saúde da rede básica de saúde (Unidades Básicas de Saúde, Secretaria Municipal de Saúde, Ambulatórios de Especialidades, Ambulatório de Saúde Mental, Vigilância Epidemiológica Municipal, Centro de Testagem e Aconselhamento, Serviço de Atendimento Especializado em DST/AIDS e um Centro de Atenção Psicossocial) do Município de São José do Rio Preto e da Direção Regional de Saúde - DIR XXII, com enfermeiros em seu quadro de pessoal, no período de dezembro/2000 e janeiro/2001. A escolha teve por base o número significativo de enfermeiros atuantes na rede básica de saúde desse município.

Ética: a execução do projeto foi autorizada pela Secretaria Municipal de Saúde de São José do Rio Preto e Direção Regional de Saúde - XXII e aprovada pelo Comitê de Ética e Pesquisa da Escola de Enfermagem de Ribeirão Preto - EERP/USP.

População: constitui-se de 80 enfermeiros que prestam assistência direta e/ou indireta na rede básica de Saúde do município e na DIR XXII. Participaram 73, pois 4 estavam em férias, 1 estava em licença-saúde e 2 não quiseram participar.

Coleta dos dados: foi feita por meio de dois instrumentos, criados pela OPAS/OMS (1997):

A - Pontos de vista sobre depressão. O questionário $A$ é uma escala com 10 afirmativas que apontam diferentes pontos de vista sobre a depressão, com graduação de 0 (desacordo) até 9 (acordo). Contém ainda informações de identificação do sujeito e do seu contexto de trabalho.

B - Conhecimento do enfermeiro sobre depressão. O questionário $B$ constitui-se de 12 questões sobre conceito, diagnóstico e tratamento da depressão, cada uma com cinco alternativas de respostas.
Os dados foram coletados por meio desses 2 questionários estruturados e auto-respondidos pelos 73 sujeitos, devidamente esclarecidos e concordes.

Análise: realizou-se a análise quantitativa descritiva dos dados, seguida de reflexão qualitativa sobre esses resultados.

\section{RESULTADOS E DISCUSSÃO}

Os participantes do estudo, na sua maioria (70 sujeitos), eram do sexo feminino, possuíam tempo de formado de 01 a 30 anos. O tempo no serviço atual variou de menos de 1 a 27 anos. Interessante notar que 23 enfermeiros $(36,4 \%)$ trabalham no mesmo local há mais de 10 anos.

Os resultados mostraram uma correlação linear positiva existente entre o tempo no serviço atual e o tempo de formado. Em sua maioria, quanto maior o tempo de formado, maior era o tempo no serviço atual.

A relação do tempo no serviço atual com o tempo total de formado indica que 1 em cada 4 entrevistados dedicou mais de $\mathbf{8 5} \%$ do tempo de formado no serviço atual, ou seja, quase não existe mobilidade.

Quanto ao interesse pela área de saúde mental (Figura 1), a menção de alto interesse na área de saúde mental (35,8\%) não é coerente com a informação sobre sua participação em cursos na área de saúde mental, pois, apesar da afirmação do alto interesse, 83,6\% não participaram de nenhum curso na área de saúde mental, nos últimos três anos.

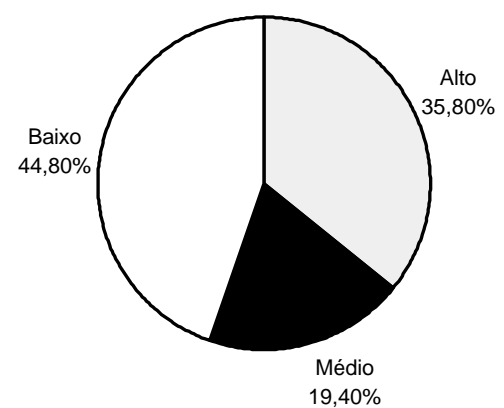

Figura 1 - Distribuição dos entrevistados, segundo seu interesse pela área de saúde mental

O baixo interesse por cursos de saúde mental indica que a maioria dos sujeitos não tem, de fato, interesse pela área de saúde mental, sugerindo reflexões a respeito das possíveis dificuldades do enfermeiro em lidar com essa área ou até em lidar com as suas próprias emoções. 
Por outro lado, o processo de formação acadêmica não tem privilegiado aspectos que possibilitem 0 fortalecimento emocional dos futuros profissionais de saúde, num nítido movimento de desconsideração que os agravos produzidos pelas situações ansiogênicas podem acarretar, aos quais os alunos estão continuamente expostos. Ao contrário, a postura dos profissionais e educadores com quem os alunos interagem, ao longo dos cursos na área da saúde, pouco os estimula a considerarem o que pensam e, principalmente, o que sentem quando se vêem confrontados com a vulnerabilidade humana ${ }^{(13)}$.

Os enfermeiros informaram que entre 20 a $50 \%$ das pessoas atendidas por eles têm depressão. Entretanto, apenas $17,8 \%$ encaminharam $10 \%$ dos casos e $15 \%$ encaminharam menos de $5 \%$ dos casos identificados. Observou-se que 23 enfermeiros não responderam a essas questões. A ausência de respostas de $31 \%$ dos enfermeiros sobre pacientes deprimidos, atendidos por eles, leva a supor que esses profissionais não têm estado em contato direto com o paciente.

Entre os fatores responsáveis pela deficiência no diagnóstico e tratamento de pacientes deprimidos (falta de destreza clínica, limitação no tempo da consulta para escutar o paciente, falta de apoio especializado para o manejo e referência de pacientes com problemas complexos) é citada pela OPAS/OMS ${ }^{(1)}$ a falta de conhecimento adequado dos profissionais sobre depressão.

A - Apresentação e discussão dos resultados dos pontos de vista sobre depressão

A Escala A identifica os pontos de vista do sujeito sobre depressão. Os trechos entre aspas são as 10 afirmativas analisadas.

A Tabela 1 mostra quatro questões com alta concentração na concordância máxima. A no 8 contém 51 enfermeiros que dela discordam totalmente: é perda de tempo atender pacientes deprimidos, e, na n 3, 31 afirmam que o paciente deprimido irrita. Esses resultados refletem incoerência e discordância nas questões relativas ao atendimento para a pessoa deprimida.

Tabela 1 - Distribuição dos pontos de vista dos enfermeiros sobre depressão, por questão

\begin{tabular}{|c|c|c|c|c|c|c|c|c|c|c|c|c|c|c|c|}
\hline \multirow{2}{*}{ Questões } & \multicolumn{4}{|c|}{ DESACORDO } & \multicolumn{5}{|c|}{ INDECISOS } & \multicolumn{4}{|c|}{ ACORDO } & \multirow{2}{*}{$\begin{array}{c}\text { Sem } \\
\text { Res- } \\
\text { posta }\end{array}$} & \multirow[t]{2}{*}{ Total } \\
\hline & $\mathbf{0}$ & 1 & 2 & Subtotal & 3 & 4 & 5 & 6 & Subtotal & 7 & 8 & 9 & Subtotal & & \\
\hline A 01 & 1 & 1 & - & 2 & 2 & 3 & 14 & 11 & 30 & 18 & 14 & 9 & 41 & - & 73 \\
\hline A 02 & 21 & 7 & 7 & 35 & 4 & 6 & 7 & 7 & 24 & 4 & 3 & 7 & 14 & - & 73 \\
\hline A 03 & 31 & 8 & 12 & 51 & 6 & 3 & 7 & 3 & 19 & 2 & - & - & 2 & 1 & 73 \\
\hline А 04 & 26 & 5 & 7 & 38 & 6 & 5 & 6 & 4 & 21 & 8 & 3 & 2 & 13 & 1 & 73 \\
\hline A 05 & 9 & 4 & 11 & 24 & 8 & 12 & 21 & 2 & 43 & 3 & 2 & 1 & 6 & - & 73 \\
\hline A 06 & 4 & 3 & 4 & 11 & 5 & 4 & 8 & 7 & 24 & 11 & 11 & 15 & 37 & 1 & 73 \\
\hline A 07 & 1 & 1 & 1 & 3 & 4 & 4 & 6 & 6 & 20 & 17 & 16 & 17 & 50 & - & 73 \\
\hline A 08 & 51 & 10 & 4 & 65 & 3 & 1 & - & 1 & 5 & 2 & - & 1 & 3 & - & 73 \\
\hline A 09 & 1 & 1 & - & 2 & 3 & 2 & 5 & 8 & 18 & 14 & 14 & 25 & 53 & - & 73 \\
\hline A 10 & 28 & 12 & 5 & 45 & 7 & 2 & 9 & 5 & 23 & 3 & - & 2 & 5 & - & 73 \\
\hline
\end{tabular}

Respostas corretas

A partir das respostas individuais dos sujeitos, de acordo com o enunciado de cada questão, da escala A, os pontos de vista de vista sobre depressão refletem mais claramente essas discordâncias.

Sessenta e seis sujeitos $(90,4 \%)$ concordam que o enfermeiro tem condições de detectar pacientes deprimidos no seu dia-a-dia (médio a completo acordo). É de se esperar que, na formação básica do enfermeiro, ele aprenda minimamente os conceitos sobre o quadro depressivo.

Por outro lado, 35 (47,9\%) não entendem que deprimir-se é um modo utilizado pelas pessoas frágeis para enfrentarem as dificuldades da vida, demonstrando possivelmente falta de informação ou preconceito do profissional.

A maioria dos enfermeiros $(69,9 \%)$ refere que não se irrita com pacientes deprimidos. Tal afirmação pode indicar a falta de vivência com a depressão na prática profissional e na sua formação. O paciente deprimido apresenta algumas dificuldades para sua assistência, mostrando apatia, irritação e, com isso, muitas vezes, faz-se 
cansativo, demandando muita energia e disposição do profissional.

Apesar de 38 (52\%) não concordarem que a depressão é uma forma de ser, difícil de ser modificada, 21 (28,7\%) mostraram-se indecisos. A indecisão pode representar falta de informação do profissional.

$\mathrm{Na}$ afirmativa trabalhar com pacientes deprimidos é uma tarefa agradável, a maioria, 43 (58,9\%), está indecisa, podendo representar uma parcela dos que não querem se comprometer, ou que não possuem experiência (formação e prática profissional) com paciente que apresenta esse tipo de patologia.

Observou-se que 48\% não apresenta uma atitude favorável à assistência em nível primário aos pacientes depressivos. Os $20,8 \%$ que concordam totalmente, apresentam uma faixa de profissionais bem esclarecida, atualizada e que poderá ser veículo de transformação da prática.

$\mathrm{Na}$ contribuição do enfermeiro para diminuir o risco de suicídio de pacientes deprimidos e quanto ao atendimento do paciente deprimido ser uma perda de tempo, houve concordância. Apesar disso, faltam elementos para avaliar a profundidade das respostas a essas questões.

A maioria $(72,6 \%)$ concorda com a contribuição do enfermeiro para melhorar a adesão ao tratamento com antidepressivos. Essa alta afirmação pode trazer uma oculta submissão do enfermeiro, principalmente em relação ao médico, ou seja, que a assistência de enfermagem se resume a essa contribuição.

A enfermagem pode não perceber e não assumir que tem um papel importante na assistência ao paciente deprimido e que tem estado muito distante do paciente que apresenta problemas emocionais. É pertinente lembrar a resistência que as pessoas têm em trabalhar com a doença mental, pelas dificuldades em lidar com as suas próprias emoções e sentimentos.

Os resultados em que 31,4\% têm dúvidas e 38.2\% concordaram que as pessoas deprimidas não procuram curarse, podem indicar uma visão de que não é preciso investir no paciente deprimido, pois este não procura curar-se, revelando, também, a falta de responsabilidade do serviço pela cura (visão manicomial da psiquiatria).

Nos resultados gerais das respostas apresentados na Tabela 2, verificam-se 44 enfermeiros $(65,8 \%)$ com escores entre 6,1 e 8,1 . Portanto, seu ponto de vista está de acordo com o que se esperava nas questões. Houve 29 respostas intermediárias e nenhum enfermeiro apresentou escores menores do que 3,9.
Tabela 2 - Distribuição dos resultados gerais (escores) dos pontos de vista sobre depressão entre enfermeiros

\begin{tabular}{cccc}
\hline ESCORES & $\begin{array}{c}\text { PONTOS } \\
\text { DE VISTA }\end{array}$ & $\begin{array}{c}\text { FREQÜÊNCIA } \\
\text { (NN) }\end{array}$ & $\begin{array}{c}\text { FREQÜÊNCIA } \\
(\%)\end{array}$ \\
\hline 0 a 2,9 & Baixo & - & - \\
3,0 a 4,4 & & 2 & 2,7 \\
4,5 a 6,0 & Médio & 27 & 37,0 \\
6,1 a 7,4 & & 37 & 50,7 \\
7,5 a 9,0 & Alto & 7 & 9,6 \\
\hline Total & & $\mathbf{7 3}$ & $\mathbf{1 0 0 , 0}$ \\
\hline
\end{tabular}

B - Apresentação e discussão dos resultados do conhecimento do enfermeiro sobre depressão (b)

Com os resultados obtidos no questionário $(\mathrm{B})$ de conhecimento dos enfermeiros sobre depressão, concluímos que a maioria (42 enfermeiros-57,6\%) tem médio conhecimento sobre depressão, mais de $1 / 3$ (32.4\%) tem ótimo nível de conhecimento sobre depressão e apenas $8,2 \%$ dos enfermeiros têm pouco conhecimento sobre depressão.

Entretanto, nos resultados das respostas por questão, destacada entre aspas, a avaliação do conhecimento dos enfermeiros mostrou diferenças, conforme se observa na Tabela 3.

Em relação à questão sobre qual a conduta do enfermeiro com paciente supostamente deprimido, 34 (46,6\%) apenas informariam ao médico. Esse posicionamento desperta reflexões sobre a submissão profissional, a desinformação ou a falta de formação adequada do profissional.

Tabela 3 - Distribuição dos resultados do conhecimento sobre depressão (B), nas 12 questões respondidas pelos 73 enfermeiros

\begin{tabular}{cccccccc}
\hline Questões & $\mathbf{1}$ & $\mathbf{2}$ & $\mathbf{3}$ & $\mathbf{4}$ & $\mathbf{5}$ & $\begin{array}{c}\text { Sem } \\
\text { resposta }\end{array}$ & TOTAL \\
\hline B01 & 12 & - & 6 & 2 & 44 & 9 & 73 \\
B02 & - & 59 & 4 & 9 & - & 1 & 73 \\
B03 & 34 & - & - & 30 & 8 & 1 & 73 \\
B04 & 3 & 1 & 9 & 23 & 28 & 9 & 73 \\
B05 & - & 25 & 9 & 8 & 25 & 6 & 73 \\
B06 & 10 & 3 & 1 & 57 & 1 & 1 & 73 \\
B07 & 3 & 8 & - & 47 & 12 & 3 & 73 \\
B08 & 18 & 1 & 2 & 37 & 5 & 10 & 73 \\
B09 & 1 & 1 & - & 3 & 66 & 2 & 73 \\
B10 & 28 & - & - & - & 44 & 1 & 73 \\
B11 & 7 & - & - & 4 & 60 & 2 & 73 \\
B12 & 1 & 2 & 5 & 1 & 64 & - & 73 \\
\hline
\end{tabular}

Respostas corretas 
Quanto ao tratamento médico que poderia servir para melhorar o quadro clínico do paciente deprimido, 12,3\% não informaram e $34,4 \%$ indicaram várias condutas medicamentosas. Essas ausências de respostas e a hipermedicalização podem ser entendidas como decorrentes da falta de conhecimento sobre depressão.

Para o tipo de psicoterapia, visando melhorar o quadro clínico da depressão, a maioria dos enfermeiros equivocouse, com 25 opções pela terapia breve (34,2\%) e 25 pela psicanálise $(34,2 \%)$. Esse equívoco parece denotar o desconhecimento do enfermeiro de seu papel de terapeuta e quais os tipos de terapia utilizados pelos diversos profissionais da área (interpessoal e cognitiva).

A associação do suicídio à depressão é de conhecimento de $91,8 \%$ enfermeiros. Sabendo disso, era de se esperar que o enfermeiro tivesse atitudes profissionais mais favoráveis ao acolhimento do paciente, ao encaminhamento adequado e condutas de ajuda.

Apesar de a maioria $(50,7 \%)$ ter assinalado a resposta esperada quanto aos efeitos secundários, encontrados nos pacientes que tomam antidepressivos tricíclicos, 10 não responderam. Durante a coleta dos dados, alguns sujeitos relataram verbalmente que não tinham conhecimento sobre os efeitos dos antidepressivos. Se o paciente deprimido busca outros serviços que não o especializado em saúde mental, inclusive as unidades básicas de saúde, que tipo de cuidado pode esperar?

Todas as opções de conduta terapêutica (ouvir atentamente sua queixa, ajudá-lo a tomar consciência do seu estado, reforçar a importância da terapia especializada e apoiá-lo em suas atividades positivas) foram consideradas pela maioria dos enfermeiros (87,7\%). No entanto, $6,8 \%$ responderam que a conduta terapêutica seria reforçar a importância da terapia especializada, excluindo qualquer atividade da enfermagem, podendo evidenciar a sua exclusão, a submissão profissional da enfermagem e a falta de conhecimento.

\section{CONSIDERAÇÕES FINAIS}

A preocupação central desse estudo esteve dirigida para a assistência ao paciente deprimido que busca ajuda na rede básica de saúde (atenção primária) e para o enfermeiro que é um dos profissionais que prestam assistência a esse cliente.

Em conseqüência, delineamos como objetivo a identificação do enfermeiro com a depressão, seus pontos de vista e seu conhecimento.

No decorrer deste trabalho, revelaram-se as implicações desses pontos de vista e desse conhecimento para a prática profissional do enfermeiro.

Os resultados mostraram que os enfermeiros não estão em contato direto com o portador de transtorno mental no seu trabalho, não sabem identificar pacientes com sintomatologia depressiva, assim como não observam esses indicadores nos pacientes por eles atendidos. Muitos não entendem que seja sua tarefa fazer essa identificação, eximindo-se da responsabilidade de identificar ou de manejar terapeuticamente o portador desse transtorno. Quando identificam os casos, nem todos os encaminham para tratamento especializado, e muitos consideram que sua tarefa limita-se à orientação medicamentosa, para melhorar a adesão ao tratamento.

Dos resultados, destaca-se ainda que, embora, no geral, o conhecimento do enfermeiro seja médio, percebe-se a falta de informações atualizadas sobre terapêuticas e sobre a possibilidade de cura de certos transtornos mentais como a depressão. A falta de responsabilidade da rede básica de saúde com esses pacientes traduz a visão manicomial, permeando esses serviços.

A identificação dos pontos de vista e do conhecimento do enfermeiro sobre depressão trouxe um diagnóstico valioso, possibilitando o encontro das necessidades e dos aspectos que precisam receber atenção nas ações em saúde mental, principalmente no que se refere à assistência de enfermagem ao portador de sintomatologia depressiva, na rede de atenção primária. Sugere que o grupo de enfermeiros desta amostra não realiza a assistência de enfermagem adequada ao paciente deprimido, necessitando de capacitação profissional para lidar com ações em saúde mental, no desempenho de suas tarefas profissionais.

A falta de conhecimento adequado do enfermeiro da rede básica de saúde para a assistência em saúde mental sugere reflexões acerca da possível relação entre as ações de enfermagem com sua formação profissional (graduação). Os resultados sugerem também que pode haver dificuldades em lidar com suas próprias emoções e evidências de submissão profissional. Todos esses aspectos podem e devem ser focalizados durante os cursos de graduação, de educação continuada, atualizações e outros aperfeiçoamentos. 


\section{Esperamos que este estudo seja uma contribuição}

para a assistência e para o ensino de enfermagem, podendo fomentar, sensibilizar e conscientizar docentes, profissionais e graduandos de enfermagem sobre a importância da atuação transformadora da prática e do ensino, em benefício do portador de transtorno mental.

\section{REFERÊNCIAS BIBLIOGRÁFICAS}

1. OPAS/OMS. Programa de Salud Mental, División de Promócion de Salud. Modelo para la capacitación de la enfermeria general en al identificación y manejo de los transtornos afectivos. Generalista I; 1997.

2. Montgomery S. Confrontando la depression. Guía del médico. New York: Pfizer Internacional; 1997.

3. Dalgalarrondo P. Psicopatologia e semiologia dos transtornos mentais. Porto Alegre: Artes Médicas; 2000.

4. Kaplan HI, Sadock BJ. Tratado de Psiquiatria. Porto Alegre: ARTMED;1997.

5. Lafer B, Almeida OP, Fraguas G Jr, Miguel EC. Depressão no ciclo da vida. Porto Alegre: Artes Médicas; 2000.

6. Willians A, Straser PB. Depression in the workplace: impact on employees. Oficial Journal of the American Association Occupational Health Nurses 1999; 47(11):526-39.

7. DSM - IV. Manual Diagnóstico e Estatístico de Transtornos Mentais. Porto Alegre: Artes Médicas; 1994.

8. CID - 10 Classificação Internacional das doenças e problemas relacionados à saúde. São Paulo: EDUSP; 1997. 9. Taylor CM. Fundamentos de enfermagem psiquiatrica de Mereness. Porto Alegre: Artes Médicas; 1992.

10. Travelbee J. Intervención en enfermeria psiquiátrica. Colombia: Carvajal; 1982.

11-Minzoni MA, Alencastre MB, Goulart MCS, Paciencia E, Rodrigues ARF, Silva JN, et al. Enfermagem em saúde mental e psiquiátrica: a busca de uma posição. Enfermagem em Novas Dimensões 1977; 3(6):350-5.

12. Pasquali L. Teoria e métodos de medida do comportamento. Brasília: MEC-SEDIA/INEP-LABPAM; 1996.

13. Esperidião E. Holismo só na teoria: a trama dos sentimentos do acadêmico de enfermagem sobre sua formação. [Dissertação]. Ribeirão Preto (SP): Escola de Enfermagem de Ribeirão Preto/USP; 2001. 